\title{
Molecular cloning of SLC35D3 and analysis of its role during porcine intramuscular preadipocyte differentiation
}

Wentong $\mathrm{Li}^{1,2 \dagger}{ }^{+}$, Keliang $\mathrm{Wu}^{3 \dagger}$, Ying Liư ${ }^{2}$, Yalan Yang ${ }^{4}$, Wenwen Wang ${ }^{1}$, Xiuxiu Li ${ }^{1}$, Yanmin Zhang ${ }^{2}$, Qin Zhang ${ }^{1}$, Rong Zhou ${ }^{2^{*}}$ and Hui Tang ${ }^{1^{*}}$ (D)

\begin{abstract}
Background: Solute carrier family 35 (SLC35) is one of a large number of membrane transporter protein families. Member D3 of this family is thought to be involved in adipose deposition and metabolic control.

Results: We obtained 2238 bp cDNA of porcine SLC35D3, it contains a 1272 bp ORF, encoding a 423 amino acid polypeptide, and a 966 bp 3' UTR. BLAST results revealed that the amino acid sequence of porcine SLC35D3 had the closest phylogenetic relationship with members of the genus Ovis aries. Further bioinformatics analysis showed that the SLC35D3 protein contains 8 transmembrane domains, and that there is no signal peptide structure. The secondary structure of the protein mainly contains $37.12 \%$ a-helixes, $7.8 \%$ in $\beta$-folds, and $33.57 \%$ random coils. mRNA expression analysis showed that SLC35D3 is expressed in lung, liver, heart, spleen, kidney, longissimus dorsi muscle (LDM), leaf fat (LF), and subcutaneous adipose tissue (SAT). To examine the effects of SLC35D3 expression on fat synthesis and catabolism, SLC35D3-siRNA was transfected into cultured intramuscular adipocytes. SLC35D3 silenced cells showed increased expression of genes related to fat synthesis, and increased deposition of intramuscular fat (IMF), abundance of lipid droplets, and the level of free fatty acid (FFA) in the culture medium. In contrast, the siRNA decreased the expression genes involved in fat catabolism.

Conclusions: Our results demonstrate that silenced SLC35D3 results in increased adipogenic processes in pig intramuscular adipocytes. These data represent the first exploration of SLC35D3 expression in swine, and provide valuable insights into the functions of SLC35D3 in adipocyte differentiation.
\end{abstract}

Keywords: SLC35D3, cDNA clone, Sequence characteristics, Tissue expression, Preadipocytes

\section{Background}

Pigs have long served as models in biomedical research because of their similarity to humans with regard to body size, physiological conditions, eating patterns, and fat deposition [1-4]. Pig breeds do vary in fat deposition and are characterized by differences in intramuscular fat content and backfat thickness. Some indigenous Chinese

\footnotetext{
* Correspondence: zhourong03@caas.cn; tanghui@sdau.edu.cn

${ }^{+}$Wentong Li and Keliang Wu contributed equally to this work.

${ }^{2}$ The State Key Laboratory of Animal Nutrition, Institute of Animal Science, Chinese Academy of Agricultural Sciences, Beijing 100193, People's Republic of China

'Shandong Provincial Key Laboratory of Animal Biotechnology and Disease Control and Prevention, College of Animal Science and Technology,

Shandong Agricultural University, 61 Daizong Street, Tai'an 271018, People's Republic of China

Full list of author information is available at the end of the article
}

breeds, such as the Yimeng Black pig, exhibit particularly high body fat mass $[5,6]$. Excessive fat deposition, which can result in obesity and disorders of energy metabolism in humans, is an important predictor of metabolic abnormalities [7]. Thus, the pig is an ideal model for the study of obesity and metabolic syndrome (MetS) $[8,9]$.

Obesity is a complex disease, it is influenced by genetic, environmental, and phenotypic factors [10-12], but the underlying mechanisms are not well understood [13]. Obesity is mainly determined by genetic differences [14], thus identification of the genes involved in fat deposition is of great interest. The solute carrier (SLC) group is the second largest group of membrane transport proteins, with more than 400 members in more than 60 families [15]. These proteins participate in 
numerous physiological processes including the transporting of inorganic ions, amino acids, sugars, lipids, neurotransmitters, and drugs [15]. Human SLC35D3 is associated with fat deposition and is a candidate gene for MetS [16]. SLC35D3 is also involved in the biogenesis of platelet dense granules, and its expression in the brain is limited to the expression of dopamine receptor D1, though not receptor D2 [16-18]. Other research indicates that SLC35D3 is an important regulator of tissue-specific autophagy [19]. SLC35D3 therefore offers an opportunity to understand the mechanisms of fat deposition, and may lead to therapeutic interventions for obesity.

In this study, we cloned the SLC35D3 CDS from the Yimeng Black pig, analyzed its amino acid sequence, and studied its expression in selected tissues and organs. We then transfected cultured intramuscular adipocytes with an SLC35D3-siRNA and examined the cells for changes in fatty acid metabolism. The results establish a foundation for understanding the function of the SLC35D3 gene in pigs.

\section{Results}

\section{Cloning and sequence analysis of the SLC35D3 gene}

Although the sequence of the porcine SLC35D3 was predicted and annotated within the Sus scrofa genome (NC_010443.4), a cDNA version has not yet been cloned. To obtain a full-length cDNA, we designed PCR primers using the annotated sequence, and used total RNA extracted from the liver tissue of a Yimeng Black pig as a PCR template. The PCR product was cloned and sequenced, the fragment (2238 bp) (KY631756.1) contains a $1272 \mathrm{bp}$ CDS encoding 423 amino acid residues, and a 966 bp 3' untranslated region (UTR). The molecular weight of the corresponding porcine SLC35D3 protein is $44,653.9 \mathrm{Da}$ and the isoelectric point is 6.94. Three potential O-glycosylation sites and 42 phosphorylation sites were found using functional site prediction algorithms. SLC35D3 does not appear to possess a signal peptide structure and is therefore unlikely to be secreted from the cell. In the predicted secondary structure, $37.12 \%$ of the amino acids participate in $\alpha$ helixes, $7.8 \%$ in $\beta$-folds, and $33.57 \%$ in random coils (Fig. 1a). Eight transmembrane domains were predicted by TMHMM analysis (Fig. 1e). Analysis using ProtScale suggests that the protein has a distinct hydrophobic region (Fig. 1b). To determine the level of amino acid conservation in homologous proteins, a multiple sequence alignment analysis was performed (Fig. 1d), revealing highly conserved regions. SWISS-MODEL was used to predict the 3D structure (Fig. 1c). Homology modeling suggests that the structure of porcine SLC35D3 is not highly homologous to human 5i20.1.A (PDB: D7A5Q8). Together, these results provide a foundation for further study of the relationship between structure and function. Finally, we used neighbor-joining to construct a phylogenetic tree from the SLC35D3 protein sequence of the Yimeng Black pig and other mammals (Fig. 1f). SLC35D3 from the Yimeng Black pig has the highest homology to members of the genus Ovis aries.

\section{Expression of $S L C 35 D 3$ in porcine tissues from different breeds and ages}

SLC35D3 mRNA levels in tissues from 180-day old Yorkshire pigs were quantified by RT-qPCR and normalized using $18 S$ RNAs (Fig. 2a). mRNA was isolated from liver, kidney, lung, heart, spleen, LDM, LF, and SAT. SLC35D3 expression was high in lung, LF, LDM, and SAT, suggesting that SLC35D3 may play an important role in adipose deposition.

We also compared SLC35D3 expression in the LDM in Yorkshire and Yimeng Black pigs. SLC35D3 expression was higher in Yorkshire 7-day old pigs than in 180-day old pigs (Fig. 2b); between the 180-day old Yorkshire and Yimeng Black pigs, expression was higher in the Yorkshire breed (Fig. 2c).

\section{Expression profile of SLC35D3 during intramuscular preadipocyte differentiation}

We performed RT-qPCR to monitor mRNA expression of SLC35D3 and the marker genes PPARY, FASN, and FABP4, in cultured preadipocytes that were undergoing differentiation. Cells were cultured for 2 days after reaching a density of $90 \%$, when they had reached confluency, then were induced to differentiate. Cells were assayed $0,2,4,6$, and 8 days after the induction of differentiation. SLC35D3 mRNA expression gradually increased to its maximum level at day 4 , and then decreased thereafter (Fig. 2e). Lipid droplet accumulation increased throughout the 8-day experiment (Fig. 2d).

\section{Silencing of SLC35D3 expression promotes adipogenesis during differentiation of porcine intramuscular preadipocytes}

The inhibition efficiency of four candidate siRNAs (siRNA 1-4) was $28,41,50$, and $24 \%$ respectively; the most effective, siRNA3, was used to inhibit the expression of SLC35D3 gene during intramuscular adipocytes differentiation. As demonstrated by Oil Red O staining of SLC35D3 silenced porcine intramuscular preadipocytes, adipogenesis was greatly increased over control cells at 8 days post induction (Fig. 3a); the level of free fatty acid (FFA) released into the culture medium also increased (Fig. 3b). As expected, relative expression of SLC35D3 decreased significantly in cells transfected with siRNA3 (Fig. 4). RT-qPCR was also used to detect the 


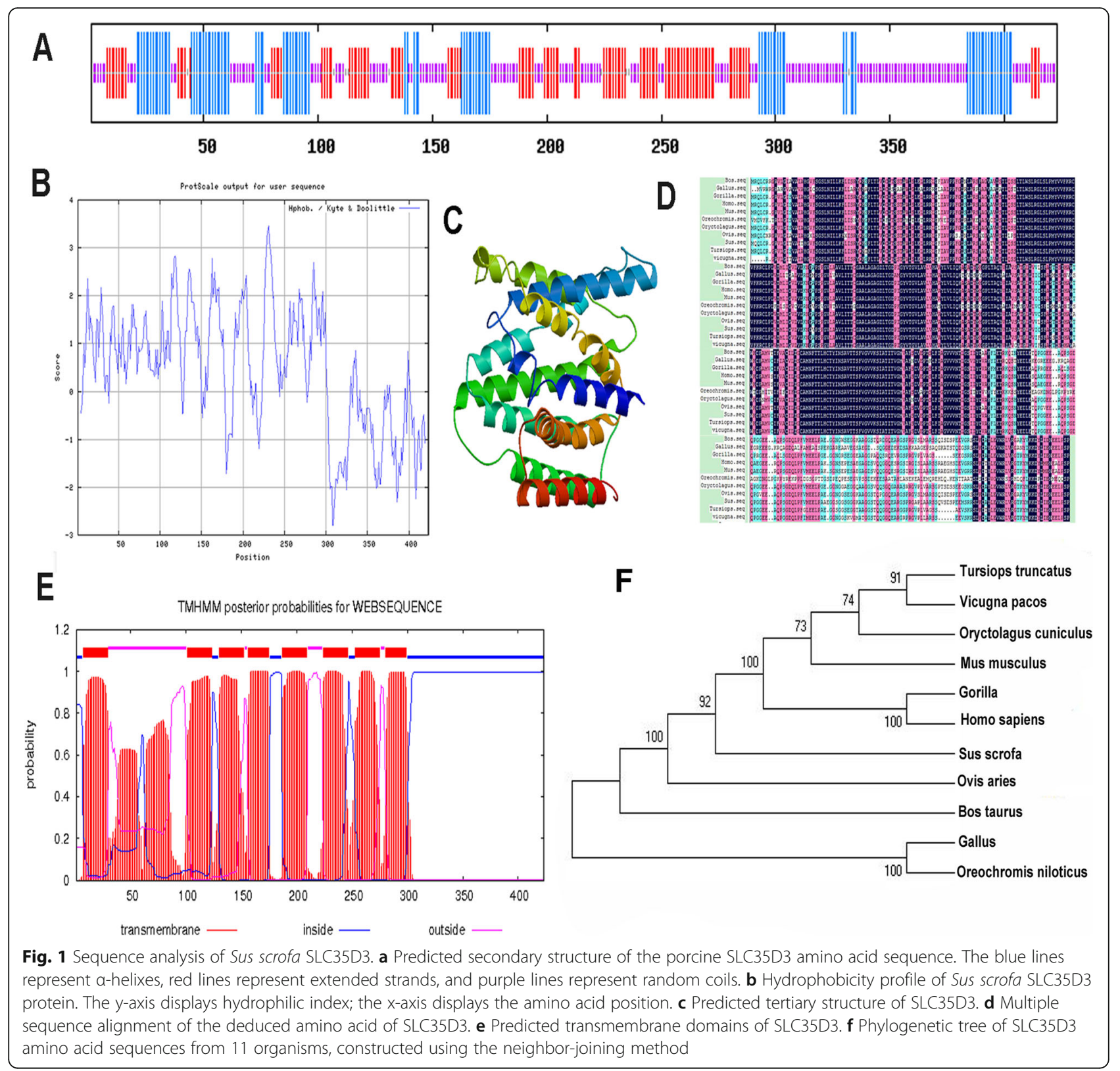

expression of five genes related to fat synthesis and catabolism (Fig. 4). Decreased expression was observed for Sirt1 and ATGL at one or more time points. In mature adipocytes, Sirt1 promotes fat mobilization through repression of PPARY [20]. ATGL is expressed in many tissues, particularly adipocytes, where it promotes both basal and stimulated lipolysis [21]. In contrast, increased expression was observed for $P P A R \gamma, C / E B P \alpha$, and $a P 2$, although the differences were not significant for $C / E B P \alpha$. $P P A R Y$ is expressed in white and brown adipose tissue, though it's expressed most highly in adipocytes and plays a key role in the regulation of adipogenesis, energy balance, and lipid biosynthesis [22]. C/EBP $\alpha$ is primarily expressed in fat, it is a key regulator at the adipogenic initiation stage, and it synergistically triggers adipocytespecific gene expression with PPARY after the growth arrest stage [23]. $a P 2$ is actively secreted from adipocytes, and is regulated by fasting- and lipolysis-related signals; circulating $a P 2$ levels are markedly elevated in obese mice and humans [24]. Overall, these results suggest that interference with $S L C 35 D 3$ gene expression promotes porcine intramuscular preadipocyte differentiation. We speculate that SLC35D3 can inhibit the adipogenic process in porcine intramuscular adipocytes.

\section{Discussion}

SLC35D3, a recently discovered gene, is involved in metabolic control, and is a candidate gene for MetS [16]. 

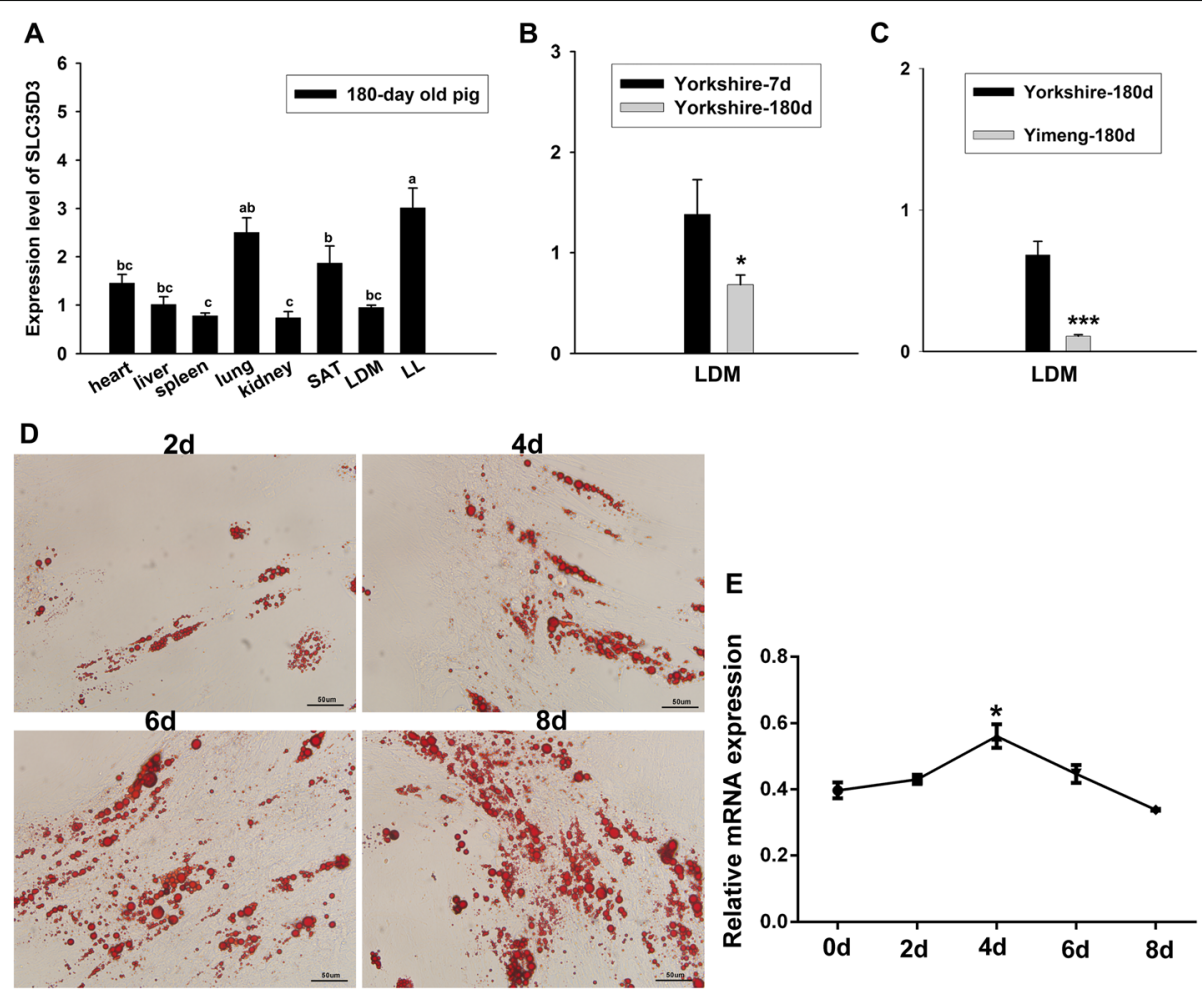

Fig. 2 Expression profile in porcine tissue distribution at different stages, breeds and during intramuscular preadipocyte differentiation. a Expression of SLC35D3 mRNA in porcine tissues from 180-day-old Yorkshire pigs. 185 was used as an internal control. b SLC35D3 mRNA expression levels in LDM from 7-day and 180-day old Yorkshire pigs. 185 was used as an internal control. c SLC35D3 mRNA expression levels in LDM from 180-day old Yorkshire and Yimeng Black pigs. 185 was used as an internal control. d Oil Red O staining of porcine intramuscular adipocytes (scale bar, $50 \mu \mathrm{m}$ ). e SLC35D3 mRNA expression during intramuscular preadipocyte differentiation. GAPDH as an internal control. Data are shown as means \pm S.E.M. $n=3$

In this study we cloned, sequenced, and characterized the porcine SLC35D3 gene. The porcine SLC35D3 sequence is similar to that described in other animals. Based on predicted physicochemical properties, porcine SLC35D3 is a hydrophobic, multi-transmembrane protein. Further sequence analysis revealed the stability and conservation of SLC35D3 gene structures.

We showed that $S L C 35 D 3$ can inhibit the adipogenic process in pig intramuscular adipocytes. Our results uncover a previously unknown role of $S L C 35 D 3$ in porcine
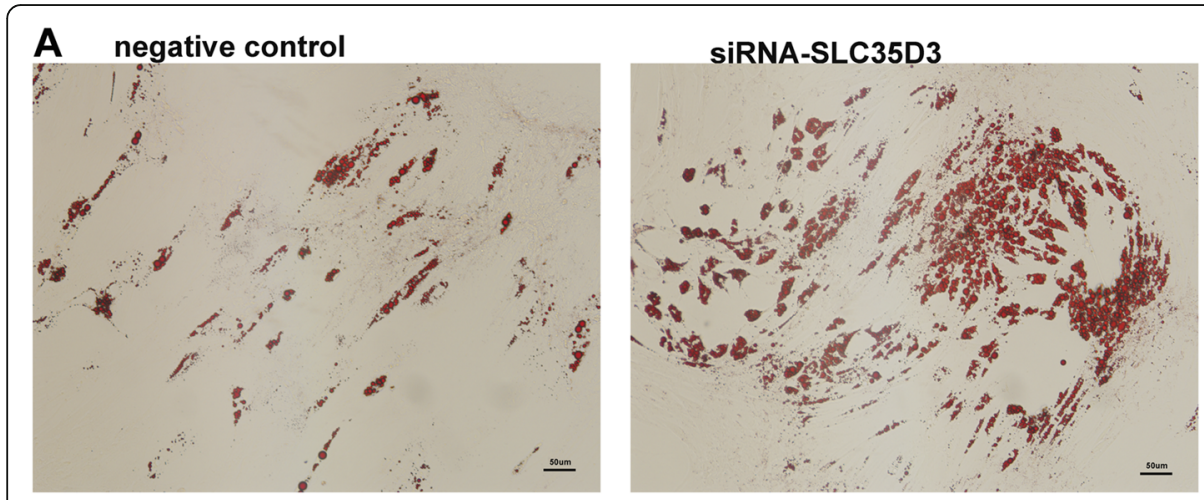

B

Fig. 3 Knockdown of SLC35D3 increased porcine intramuscular preadipocyte differentiation (a) The effects of SLC35D3 silencing on lipid droplet accumulation in intramuscular adipocytes 8 days after the induction of differentiation (scale bar, $50 \mu \mathrm{m}$ ). $\mathbf{b}$ The effects of SLC35D3 gene silencing on free fatty acid in culture medium of porcine intramuscular adipocytes. Data are shown as means \pm S.E.M. $n=3$. NC, negative control siRNA; siRNA, SLC35D3-siRNA 


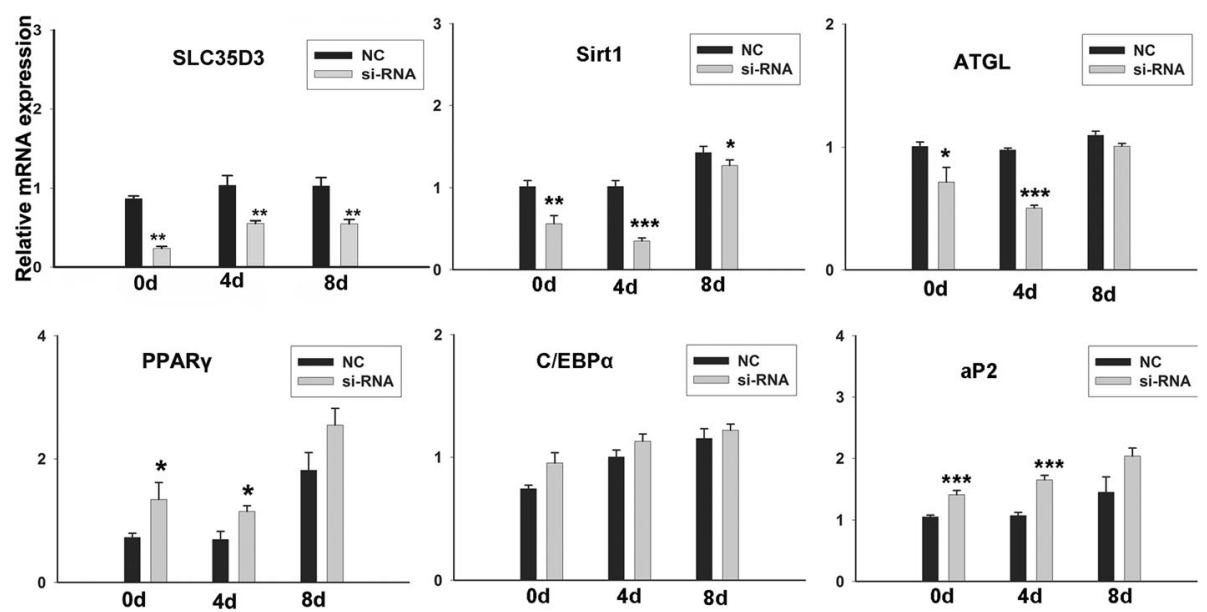

Fig. 4 mRNA expression profile of SLC35D3 and adipogenic marker genes during intramuscular adipogenesis by RT-qPCR. GAPDH is the internal control. Data are shown as means \pm S.E.M. $n=3$

adipogenesis, and suggest a fruitful area of research for improving the quality of porcine meat quality as well as its potential role in human cardiovascular diseases.

Essential in the study of mechanisms involved in fat deposition, is the study of IMF. IMF is a primary factor of meat quality, it is affected by fatty acid transport, fat anabolism, and catabolism. To date there are no studies examining expression of SLC35D3 in the various tissues of pigs based on age or breed. Our results showed that the expression levels of $S L C 35 D 3$ were high in fat and LDM tissue. In LDM, expression is higher in 7-day old piglets than in 180-day old pigs. In addition, the level of gene expression is significantly different in Yorkshire and Yimeng Black pigs. The Yorkshire pig is a typical lean meat breed, it has less subcutaneous and intramuscular fat than the Yimeng Black pig. It is still unknow whether the expression level of SLC35D3 is related to the fat deposition between the two pig breeds. During differentiation of porcine intramuscular preadipocytes, SLC35D3 expression increased from day 0 to day 4 and then declined until day 8 . This trend was also seen with the expression of the adipogenic marker genes. We therefore speculate that SLC35D3 has important functions during adipocyte differentiation, however the underlying mechanism is still unknown. Adipogenesis is a complex programmed process, during which the early adipogenic transcription factors $C / E B P \alpha$ and $P P A R \gamma$ are activated, inducing the expression of adipogenic genes. These factors also play a critical role in the terminal differentiation of adipocytes in vitro, ultimately leading to the formation of mature adipocytes [25, 26]. ATGL initiates the process of triglyceride metabolism by hydrolyzing triglycerides into diacylglycerol and fatty acids $[27,28]$. We found that reducing expression of SLC35D3 in porcine intramuscular preadipocytes resulted in siginificantly higher expression of adipogenic marker genes such as PPARy and aP2, while inhibiting the expression of lipid hydrolytic gene ATGL. However, the observed trends of $C / E B P \alpha$ were not significant. Consistent with these results, FFA levels increased in the culture medium of intramuscular preadipocytes during differentiation. These results together suggest that SLC35D3 may be a specific transcription regulatory factor during porcine intramuscular preadipocyte differentiation.

\section{Conclusions}

In summary, this is the first report describing the cDNA sequence of SLC35D3 from the Yimeng Black pig. The gene contains a $1272 \mathrm{bp}$ CDS that encodes 423 amino acids, and a 966 bp 3' UTR. The deduced amino acid sequence of SLC35D3 is highly similar (92-95\%) to homologous proteins in other mammalian species. Phylogenetic analysis shows that pig SLC35D3 has a close evolutionary relationship with the Ovis aries version of the gene. Gene expression analysis suggests that $S L C 35 D 3$ inhibits adipogenesis in pig intramuscular preadipocytes. Our results provide a basis for further studies on the function and regulation of SLC35D3.

\section{Methods}

\section{Experimental animals and sampling}

Three 180-day old Yorkshire pigs (average live weight $100 \mathrm{~kg}$; range, $99-105 \mathrm{~kg}$ ), three 7-day old Yorkshire pigs (average live weight, $1 \mathrm{~kg}$; range, $0.8-1.3 \mathrm{~kg}$ ), and three 180-day old Yimeng Black pigs (average live weight 100 $\mathrm{kg}$; range, $90-110 \mathrm{~kg}$ ) were purchased from the experiment farm at the Chinese Academy of Agricultural Sciences; all animals were female. Animals had been maintained under the same management conditions, fed the same food three times a day, and had access to water 
ad libitum. Animals selected for tissue collection were humanely euthanized by electrical stunning followed by exsanguination. Tissue samples from the liver, kidney, lung, heart, spleen, LDM, LF, and SAT were dissected from each pig, immediately frozen in liquid nitrogen, transported to the laboratory, and stored at $-80{ }^{\circ} \mathrm{C}$ until RNA extraction. Intramuscular preadipocytes were collected from the 7-day old piglets and cultured. Finally, stored at $-80{ }^{\circ} \mathrm{C}$ until use.

\section{RNA isolation and CDNA synthesis}

Total RNA was extracted from tissues and cells using TRIzol reagent (Invitrogen, Carlsbad, CA, USA). RNA concentrations were determined by absorption spectrophotometry at $260 \mathrm{~nm}$. First-strand cDNA synthesis was conducted with $2 \mu \mathrm{g}$ of purified total RNA using a RevertAid First-Strand cDNA Synthesis Kit (Thermo Scientific, Waltham, MA, USA). RNA and cDNA were stored at $-80^{\circ} \mathrm{C}$ and $-20^{\circ} \mathrm{C}$, respectively.

\section{PCR amplification of the internal fragment of SLC35D3}

Forward (F) and reverse (R) primers (Table 1) were designed with the Primer Premier 5.0 application, using porcine SLC35D3 (XM_013986971.1) as the reference gene sequence. The PCR reaction contained $1 \mu \mathrm{L}$ of cDNA, $12.5 \mu \mathrm{L}$ of $2 \times$ Es Taq Master Mix, $0.5 \mu \mathrm{L}$ of each primer, and $10.5 \mu \mathrm{L}$ of RNase-free water. PCR cycling conditions were $94{ }^{\circ} \mathrm{C}$ for $5 \mathrm{~min}$, then 34 cycles of $94{ }^{\circ} \mathrm{C}$ for $30 \mathrm{~s}, 57^{\circ} \mathrm{C}$ for $5 \mathrm{~s}, 72^{\circ} \mathrm{C}$ for $26 \mathrm{~s}$, followed by $72^{\circ} \mathrm{C}$ for $10 \mathrm{~min}$.

\section{5' RACE and 3' RACE}

First-strand cDNA synthesis was accomplished using the SMARTer RACE 5'/3' Kit (Takara, Dalian, China) according to the manufacturer's protocol. 5' RACE and 3' RACE reactions were performed by nested PCR, using the SLC35D3-specific primers GSP5/3 and NGSP5 and the universal primers UPM long and UPM short (Table 1).

All PCR products, including the internal fragment and fragments generated by $5^{\prime}$ RACE and 3' RACE, were subjected to agarose gel electrophoresis, then recovered using an agarose gel DNA Purification Kit (Tiangen, Beijing, China). The products were cloned into the pEASY-T1 vector (Trans, Beijing, China). Clones were submitted to Sangon Biotech Co., Ltd. (Shanghai, China) for nucleotide sequencing.

\section{Sequence analysis}

Molecular weight and isoelectric point were predicted using Compute pI/Mw (http://us.expasy.org/tools/pi_ tool.html). The secondary structure of the deduced amino acid sequence was predicted by SOPMA (http://
Table 1 Primer sequences and their use in this study

\begin{tabular}{|c|c|}
\hline Primer name & Primer sequence $\left(5^{\prime} \rightarrow 3^{\prime}\right)$ \\
\hline $\mathrm{F}$ & TGCACCTACATCAACTCGG \\
\hline $\mathrm{R}$ & TCATTTCTTCAGGGCTGTCT \\
\hline UPM long & $\begin{array}{l}\text { CTAATACGACTCACTATAGGGCAAGCAG } \\
\text { TGGTATCAACGCAGAGT }\end{array}$ \\
\hline UPM short & CTAATACGACTCACTATAGGGC \\
\hline GSP3 & GGTGAAGAGCATCGCCACCATCACGG \\
\hline GSP5 & CCCCTCGCCTCTTGCCCACCCTGCT \\
\hline NGSP5 & GCCTTCCCACCTCCTGACCCGCC \\
\hline SLC35D3-F & CCTCAGCCTGCCTATGTACG \\
\hline SLC35D3-R & CAGCGCTTGCTTTCTGGATG \\
\hline ATGL-F & TTGCTGTCAACCAACCACTC \\
\hline ATGL-R & TAATAGTGCTCTGAGGGCCG \\
\hline PPARY-F & AGGACTACCAAAGTGCCATCAAA \\
\hline$P P A R Y-R$ & GAGGCTTTATCCCCACAGACAC \\
\hline Sirt1-F & AACCGATGGAGAGTCCAGGT \\
\hline Sirt1-R & TACCTCAGCGCCATGGAAAA \\
\hline$a P 2-F$ & GAGCACCATAACCTTAGATGGA \\
\hline$a P 2-R$ & AAATTCTGGTAGCCGTGACA \\
\hline C/EBPa-F & CGATGCTCTTAGCTGAGTGT \\
\hline C/EBPa-R & GGTCCAAGAATTTCACCTCT \\
\hline GAPDH-F & AGGGCATCCTGGGCTACACT \\
\hline GAPDH-R & TCCACCACCCTGTTGCTGTA \\
\hline $18 S-F$ & CGTCTGCCCTATCAACTTT \\
\hline $18 S-R$ & TITCTCAGGCTCCCTCTC \\
\hline
\end{tabular}

npsa-pbil.ibcp.fr/) [29]. Phylogenetic analyses were performed using MEGA 5.1, applying the neighborjoining method [30]. Amino acid sequences from different species were aligned using DNAMAN V6 (LynnonBiosoft, Los Angeles, CA, USA) [31]. SWISSMODEL was used to model 3D protein structure [32, 33]. TMHMM Server v2.0 program (http://www.cbs.dtu. $\mathrm{dk} /$ services/TMHMM/) was used for protein transmembrane Structure analysis. ExPASy ProtScal (http://web. expasyorg/protscale/) was used for hydrophobic analysis. The signal peptide was predicted using SignalP (http:// www.cbs.dtu.dk/services/SignalP/) [34]. N-glycosylation and O-glycosylation sites were predicted using NetNGlycears 1.0 (http://www.cbs.dtu.dk/services/NetNGlyc/) and NetOGlycubles 3.1 (http://www.cbs.dtu.dk/services/ NetOGlyc/), respectively [35].

RT-qPCR for expression profile analysis Primers for SLC35D3, Sirt1 (NM_001145750.2), ATGL (EF583921.1), PPARY (NM_214379), C/EPBQ (XM_ 003127015), and aP2 (AJ555153.1) were designed using Primer Premier 5.0 (Premier Biosoft International, Palo Alto, CA). Relative mRNA levels were normalized 
against GAPDH and $18 S$ expression. The PCR reaction contained $7.2 \mu \mathrm{L}$ of $2 \times$ SYBR Premix Ex Taq (Takara, Dalian, China), $0.3 \mu \mathrm{L}$ of each primer, $1 \mu \mathrm{L}$ of cDNA, $0.3 \mu \mathrm{L}$ of Dye II, and sterile water to a final volume $15 \mu \mathrm{L}$. PCR cycling conditions were: $95^{\circ} \mathrm{C}$ for $5 \mathrm{~min}$, followed by 40 cycles at $95^{\circ} \mathrm{C}$ for $5 \mathrm{~s}$ and $60^{\circ} \mathrm{C}$ for $34 \mathrm{~s}$. Finally, a dissociation step was performed at $95^{\circ} \mathrm{C}$ for $15 \mathrm{~s}, 60^{\circ} \mathrm{C}$ for $1 \mathrm{~min}$, and $95^{\circ} \mathrm{C}$ for $15 \mathrm{~s}$. All samples were amplified in triplicate, and the mean was used for further analysis. Amplification of target genes was determined using the $2^{-\Delta \Delta \mathrm{Ct}}$ method.

\section{Isolation and culture of intramuscular preadipocytes}

LDM was collected from 7-day old piglets under aseptic conditions. Tissue samples were washed 3 times in PBS containing $1 \%$ penicillin and streptomycin, and cut into small pieces (approximately $1 \mathrm{~mm}^{3}$ ). Tissue pieces were digested in $0.1 \%$ type I collagenase (Invitrogen, Carlsbad, CA, USA) for $1 \mathrm{~h}$ at $37^{\circ} \mathrm{C}$, then filtered through 400 mesh filters. The filtrates were centrifuged for $5 \mathrm{~min}$ at $1500 \mathrm{rpm} / \mathrm{min}$. Cell pellets were washed with PBS then centrifuged again for $5 \mathrm{~min}$ at $1500 \mathrm{rpm} / \mathrm{min}$, repeat 3 times. The preadipocytes were resuspended in DMEM/ F12 containing 10\% fetal bovine serum (FBS) (Sigma, St. Louis, MO, USA) and $100 \mathrm{U} / \mathrm{mL}$ penicillin and streptomycin, then seeded into 6-well plates at a density of $5 \times 10^{4}$ cells $/ \mathrm{cm}^{2}$, and incubated at $37^{\circ} \mathrm{C}$ in a humidified $5 \% \mathrm{CO}_{2}$ atmosphere. Culture medium was changed every two days.

\section{siRNA design and transfection}

Gene-specific siRNAs (Table 2) for Sus scrofa SLC35D3 was synthesized based on our cDNA sequence; these were designed and synthesized by Gene Pharma Co., Ltd. Preadipocytes at $70-80 \%$ confluence were transfected with a negative control siRNA $(20 \mathrm{nM})$ or SLC35D3-siRNA (20 nM) using Lipofectamine 2000 in OPTI-MEM, according to the manufacturer's protocol. $48 \mathrm{~h}$ after transfection, culture medium was replaced with DMEM/F12 supplemented with 10\% FBS and

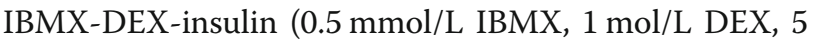

Table 2 siRNA sequences

\begin{tabular}{|c|c|}
\hline name & seqence $\left(5^{\prime} \rightarrow 3^{\prime}\right)$ \\
\hline negative control & $\begin{array}{l}\text { Sense 5'-UUCUCCGAACGUGUCACGUTT-3' } \\
\text { Antisense 5'-ACGUGACACGUUCGGAGAATT-3' }\end{array}$ \\
\hline SLC35D3-siRNA1 & $\begin{array}{l}\text { Sense 5'-UCAUCAGCCGCUACCAGUUTT-3' } \\
\text { Antisense 5'-AACUGGUAGCGGCUGAUGATT-3' }\end{array}$ \\
\hline SLC35D3-siRNA2 & $\begin{array}{l}\text { Sense 5'- GCAUCUUCGUGGCCUGUAUTT -3' } \\
\text { Antisense 5'- AUACAGGCCACGAAGAUGCTT - 3' }\end{array}$ \\
\hline SLC35D3-siRNA3 & $\begin{array}{l}\text { Sense 5'-GCCCACCUCUCUAUUCAUUTT-3' } \\
\text { Antisense 5'-AAUGAAUAGAGAGGUGGGCTT-3' }\end{array}$ \\
\hline SLC35D3-siRNA4 & $\begin{array}{l}\text { Sense 5'-GGAAGUGUGGCGGUUAGUUTT-3' } \\
\text { Antisense 5'-AACUAACCGCCACACUUCCT-3' }\end{array}$ \\
\hline
\end{tabular}

$\mathrm{mg} / \mathrm{mL}$ insulin) to induce differentiation. Cells were incubated in this medium for $48 \mathrm{~h}$, then the culture medium was changed to DMEM/F12 with $10 \%$ FBS and $5 \mathrm{mg} / \mathrm{mL}$ insulin, this medium was changed every 2 days. Total RNA was extracted from cells on days 0,4 , and 8.

\section{Oil red $O$ staining}

Oil Red O staining was conducted as described previously [36].

\section{Measurement of cellular lipid metabolites}

Preadipocytes were induced, as described above, for 8 days, then culture medium was collected and submitted to North Life Science Co., Ltd. (Beijing, China) for free fatty acid analysis. The FFA measurements were conducted according to the manufacturer's protocol provided in the free fatty acid analysis kit (Njjcbio, Nanjing, China).

\section{Statistical analysis}

Data was analyzed using the SPSS v22.0 (SPSS, Chicago, IL), and one-way analysis of variance was used to assess the significance of experimental results. All data are presented as the means \pm standard error of the mean. Differences were considered significant at a $P$ value of $\left.<0.05^{(*)},<0.01^{(* *)}\right)$, or $<0.001^{(* * *)}$.

\section{Abbreviations}

CDS: Coding sequence; FBS: Fetal bovine serum.; FFA: Free fatty acid; IMF: Intramuscular fat; LDM: Longissimus dorsi muscle; LF: Leaf fat; MetS: Metabolic syndrome; SAT: Subcutaneous adipose tissue; SLC35: Solute carrier family 35; UTR: Untranslated region

Acknowledgements

We thank the farmers at the Experiment Farm at the Chinese Academy of Agricultural Sciences for allowing us to collect the samples.

\section{Authors' contributions}

HT and RZ conceived the project and designed the experiments; $W L$ and $Y Y$ analyzed the data. XL and WW collected samples; $Y L, W L$ and $Y Z$ performed the experiments; WL wrote the manuscript; HT, QZ and KW revised the paper. All authors read and approved the final manuscript.

\section{Funding}

The study was supported by the Shandong "Double Tops" Program (SYL2017YSTD12) (study design, travel and sampling costs, manuscript preparation); the National Natural Science Foundation of China (31501931 and 31330074) (travel and sampling costs, and costs incurred analyzing and interpreting the data); the Program of New Breed Development via

Transgenic Technology (2016ZX080011-006) (travel and sampling costs); and the National Nonprofit Institute Research Grant (2018-YWF-YB-7 and Y2016JC07) (costs incurred analyzing and interpreting data and writing the manuscript). The data interpretation was also supported by the Foshan University Initiative Scientific Research Program.

\section{Availability of data and materials}

All gene sequences in this study were deposited in NCBI. SLC35D3 (KY631756.1), Sirt1 (NM_001145750.2), ATGL (EF583921.1), PPARY (NM_214379), C/EPBa (XM_003127015), and AP2 (AJ555153.1).

\section{Ethics approval and consent to participate}

All animals were treated humanely according to criteria outlined in the "Guide for the Care and Use of Laboratory Animals" published by the 
Institute of Animal Sciences, Chinese Academy of Agricultural Sciences (Beijing, China). Procedures were approved by the Animal Care and Use Committee. Pigs were slaughtered following the Animal Care Guidelines of the Ethics committee of Chinese Academy of Agricultural Sciences.

\section{Consent for publication}

Not applicable.

\section{Competing interests}

The authors declare that they have no competing interests.

\section{Author details}

${ }^{1}$ Shandong Provincial Key Laboratory of Animal Biotechnology and Disease Control and Prevention, College of Animal Science and Technology, Shandong Agricultural University, 61 Daizong Street, Tai'an 271018, People's Republic of China. ${ }^{2}$ The State Key Laboratory of Animal Nutrition, Institute of Animal Science, Chinese Academy of Agricultural Sciences, Beijing 100193, People's Republic of China. ${ }^{3}$ College of Animal Science and Technology, China Agricultural University, Beijing 100193, People's Republic of China. ${ }^{4}$ School of Life Science and Engineering, Foshan University, Foshan 528231, Guangdong, China.

\section{Received: 18 October 2018 Accepted: 4 February 2020}

Published online: 22 February 2020

\section{References}

1. Lunney JK. Advances in swine biomedical model genomics. Int J Biol Sci. 2007;3(3):179-84.

2. Ross JW, et al. Generation of an inbred miniature pig model of retinitis pigmentosa. Invest Ophthalmol Vis Sci. 2012;53(1):501-7.

3. Xing $K$, et al. Integration of transcriptome and whole genomic resequencing data to identify key genes affecting swine fat deposition. PLoS One. 2015;10(4):e0122396.

4. Stachowiak M, Szczerbal I, Switonski M. Genetics of adiposity in large animal models for human obesity-studies on pigs and dogs. Prog Mol Biol Transl Sci. 2016;140:233-70.

5. Li C, et al. Molecular microevolution and epigenetic patterns of the long non-coding gene $\mathrm{H} 19$ show its potential function in pig domestication and breed divergence. BMC Evol Biol. 2016;16:87.

6. Fang M, Andersson L. Mitochondrial diversity in European and Chinese pigs is consistent with population expansions that occurred prior to domestication. Proc Biol Sci. 2006:273(1595):1803-10.

7. Huang W, et al. Differential regulation of mRNAs and IncRNAs related to lipid metabolism in two pig breeds. Oncotarget. 2017:8(50):87539-53.

8. Bohan $A E$, et al. The proliferation and differentiation of primary pig preadipocytes is suppressed when cultures are incubated at 37 degrees Celsius compared to euthermic conditions in pigs. Adipocyte. 2014;3(4):322-32.

9. Spurlock ME, Gabler NK. The development of porcine models of obesity and the metabolic syndrome. J Nutr. 2008;138(2):397-402.

10. Speakman JR. Obesity: the integrated roles of environment and genetics. $J$ Nutr. 2004;134(8 Suppl):2090S-105S.

11. Zhang H, Wu J, Yu L. Association of GIn27Glu and Arg16Gly polymorphisms in Beta2-adrenergic receptor gene with obesity susceptibility: a metaanalysis. PLoS One. 2014;9(6):e100489.

12. de Oliveira CM, et al. Heritability of cardiovascular risk factors in a Brazilian population: Baependi heart study. BMC Med Genet. 2008;9:32.

13. Vieira-Potter VJ, et al. Disconnect between adipose tissue inflammation and cardiometabolic dysfunction in Ossabaw pigs. Obesity (Silver Spring). 2015;23:2421-9.

14. Comuzzie AG, et al. Novel genetic loci identified for the pathophysiology of childhood obesity in the Hispanic population. PLoS One. 2012;7(12):e51954.

15. Bai X, Moraes TF, Reithmeier RAF. Structural biology of solute carrier (SLC) membrane transport proteins. Mol Membr Biol. 2017;34(1-2):1-32.

16. Zhang Z, et al. Mutation of SLC35D3 causes metabolic syndrome by impairing dopamine signaling in striatal D1 neurons. PLoS Genet. 2014; 10(2):e1004124.

17. Chintala S, et al. The Slc35d3 gene, encoding an orphan nucleotide sugar transporter, regulates platelet-dense granules. Blood. 2007;109(4):1533-40.
18. Meng R, et al. SLC35D3 delivery from megakaryocyte early endosomes is required for platelet dense granule biogenesis and is differentially defective in Hermansky-Pudlak syndrome models. Blood. 2012;120(2):404-14.

19. Wei ZB, et al. SLC35D3 increases autophagic activity in midbrain dopaminergic neurons by enhancing BECN1-ATG14-PIK3C3 complex formation. Autophagy. 2016;12(7):1168-79.

20. Picard F, et al. Sirt1 promotes fat mobilization in white adipocytes by repressing PPAR-gamma. Nature. 2004;429(6993):771-6.

21. Schoiswohl G, et al. Impact of reduced ATGL-mediated adipocyte lipolysis on obesity-associated insulin resistance and inflammation in male mice. Endocrinology. 2015;156(10):3610-24.

22. Janani C, Ranjitha Kumari BD. PPAR gamma gene--a review. Diabetes Metab Syndr. 2015;9(1):46-50.

23. Ji S, Doumit ME, Hill RA. Regulation of Adipogenesis and key Adipogenic gene expression by 1, 25-Dihydroxyvitamin D in 3T3-L1 cells. PLoS One. 2015;10(6):e0126142

24. $\mathrm{Cao} \mathrm{H}$, et al. Adipocyte lipid chaperone AP2 is a secreted adipokine regulating hepatic glucose production. Cell Metab. 2013;17(5):768-78.

25. Nagai S, et al. Suppression of Fatty Acid and Triglyceride Synthesis by the Flavonoid Orientin through Decrease of C/EBPdelta Expression and Inhibition of PI3K/Akt-FOXO1 Signaling in Adipocytes. Nutrients. 2018;10(2).

26. Bouraoui L, Gutierrez J, Navarro I. Regulation of proliferation and differentiation of adipocyte precursor cells in rainbow trout (Oncorhynchus mykiss). J Endocrinol. 2008;198(3):459-69.

27. Zimmermann $\mathrm{R}$, et al. Fat mobilization in adipose tissue is promoted by adipose triglyceride lipase. Science. 2004;306(5700):1383-6.

28. Liu X, et al. Long non-coding RNA NEAT1-modulated abnormal lipolysis via ATGL drives hepatocellular carcinoma proliferation. Mol Cancer. 2018;17(1):90.

29. Geourjon C, Deleage G. SOPMA: significant improvements in protein secondary structure prediction by consensus prediction from multiple alignments. Comput Appl Biosci. 1995;11(6):681-4.

30. Biradar SS, et al. Genetic characterization of toxoplasma gondii isolates from chickens in India by GRA6 gene sequence analysis. Acta Parasitol. 2014; 59(4):666-74.

31. Wan $\mathrm{L}$, et al. Molecular cloning, structural analysis and tissue expression of protein phosphatase 3 catalytic subunit alpha isoform (PPP3CA) gene in Tianfu goat muscle. Int J Mol Sci. 2014;15(2):2346-58.

32. Kiefer $F$, et al. The SWISS-MODEL repository and associated resources. Nucleic Acids Res. 2009;37(Database issue):D387-92.

33. Arnold K, et al. The SWISS-MODEL workspace: a web-based environment for protein structure homology modelling. Bioinformatics. 2006;22(2):195-201.

34. Li $Z$, et al. Characterization of the visfatin gene and its expression pattern and effect on 3T3-L1 adipocyte differentiation in chickens. Gene. 2017;632:16-24

35. Bashir $U$, et al. Molecular detection and characterization of respiratory syncytial virus B genotypes circulating in Pakistani children. Infect Genet Evol. 2017:47:125-31.

36. Zhao C, et al. MAT2B promotes adipogenesis by modulating SAMe levels and activating AKT/ERK pathway during porcine intramuscular preadipocyte differentiation. Exp Cell Res. 2016;344(1):11-21.

\section{Publisher's Note}

Springer Nature remains neutral with regard to jurisdictional claims in published maps and institutional affiliations.

\section{Ready to submit your research? Choose BMC and benefit from:}

- fast, convenient online submission

- thorough peer review by experienced researchers in your field

- rapid publication on acceptance

- support for research data, including large and complex data types

- gold Open Access which fosters wider collaboration and increased citations

- maximum visibility for your research: over $100 \mathrm{M}$ website views per year

At BMC, research is always in progress.

Learn more biomedcentral.com/submissions 